\title{
Expression Patterns of the chgH:rfp Transgene in Response to 17a-Ethinylestradiol (EE2) Exposure in Marine Medaka Oryzias dancena
}

\author{
Yoon Kwon Nam ${ }^{1,2 *}$, Young Sun $\mathrm{Cho}^{2}$ and Dong Soo $\mathrm{Kim}^{2}$ \\ ${ }^{1}$ Center of Marine-Integrated Biomedical Technology (BK21 Plus Team), Pukyong National University, Busan 608-737, Korea \\ ${ }^{2}$ Institute of Marine Living Modified Organisms, Pukyong National University, Busan 608-737, Korea
}

\begin{abstract}
The functional utility of a transgenic marine medaka Oryzias dancena strain carrying the red fluorescent protein (RFP) gene driven by an endogenous choriogenin $\mathrm{H}(\mathrm{chgH})$ promoter was evaluated for its ability to detect waterborne 17 $\alpha$-ethinylestradiol (EE2), a synthetic estrogen derivative. The chgH:rfp transgenic marine medaka larvae showed an age-dependent tendency in the efficiency of EE2-mediated transgene expression, in which transgenic larvae older than 6 days post-hatching displayed a more effective response in their transgene expression to EE2 than did younger hatchlings. During experimental exposures to high concentrations of EE2 (200 to $1,000 \mathrm{ng} / \mathrm{L})$, the transgenic responses in the hatchlings were broadly dose- and duration-dependent. With exposures using lower doses of EE2 (25, 50 and $100 \mathrm{ng} / \mathrm{L})$, EE2-induced transgenic RFP was also observed in the transgenic larvae, although the lower doses required exposure of longer duration. Under the EE2 exposure and microscope assay conditions used in our study, transgenic marine medaka larvae exhibited a similar degree of EE2-mediated RFP phenotype expression at various salinity levels $(0,15$ and $30 \mathrm{ppt})$.
\end{abstract}

Key words: Oryzias dancena, Marine medaka, EE2, chgH:rfp-Transgenics

\section{Introduction}

EE2 (17 $\alpha$-ethinylestradiol) is an orally bioactive estrogen, which has been widely used in both human medications and livestock management activities. This synthetic hormone has become a widespread contaminant in the aquatic environment, mainly because of its high resistance to degradation, and its tendency to interfere with and/or deregulate the endocrine system of living organisms in contaminated environments (Aris et al., 2014). A number of previous studies have reported the detrimental effects of EE2 on aquatic organisms with particular emphasis on damage to the reproductive systems of bony fishes. These include the changing of the sex ratio and reproductive capability (Lange et al., 2001), changed gene expres- sion profile related to sexual maturation (Filby et al., 2007; Hirakawa et al., 2012), and alteration of male competitive courtship behavior (Lee et al., 2014). The adverse effects of EE2 on fish have not been limited to reproductive issues. EE2 has also been reported to alter the growth and differentiation patterns, innate immunity, apoptosis inhibition, and metabolomic profiles in exposed fish (Shved et al., 2008, 2009; Nadzialek et al., 2010; Teng et al., 2013). For these reasons, the development of biomarker-assisted approaches to elucidate the mechanism (s) underlying the influence of EE2 on the cellular and physiological pathways in fish is crucial to address the effects of EE2 on contaminated biota (Filby et al., 2007;
(C) 2015 The Korean Society of Fisheries and Aquatic Science This is an Open Access article distributed under the terms of the Creative Commons Attribution Non-Commercial Licens (http://creativecommons. org/licenses/by-nc/3.0/) which permits unrestricted non-commercial use, distribution, and reproduction in any medium, provided the original work is properly cited.
Received 02 September 2014; Accepted 30 October 2014

*Corresponding Author

E-mail: yoonknam@pknu.ac.kr 
Harding et al., 2013).

A number of transgenic fish models incorporating fluorescence protein reporters have been established for detecting waterborne estrogenic compounds, in which estrogen-responsive promoters were designed to drive the expression of visible fluorescent protein in specific response to exposure to exogenous estrogenic chemicals (Chen et al., 2010; Petersen et al., 2013). As an in vivo model system, such a transgenebased approach to measure environmental estrogenicity is useful since it allows the simple and non-invasive detection of the response to estrogens in living fish (Kurauchi et al., 2008; Chen et al., 2010). Furthermore, this in vivo system offers a potential platform for obtaining on-site and real-time insights into estrogen-mediated responses in fish, which facilitate the development of multivalent and integrated regimes to address the cross-talk between the modulation of estrogen-responsive genes and other physico-biochemical or behavioral changes (Lee et al., 2012a).

We recently reported the potential of a transgenic system constituting a fluorescent reporter gene driven by a choriogenin $\mathrm{H}$ promoter developed in a euryhaline marine medaka (Oryzias dancena) in detecting waterborne estrogenicity. The selected transgenic line has been shown to respond to various estrogenic chemicals (Cho et al., 2013). Marine medaka is a truly euryhaline species with the important capability of osmoregulation, in which this species displays a similar level of performance regarding survival, growth and reproduction under different salinity conditions. For this reason, if successfully developed, an estrogen-responsive transgenic system in this species could be applied to a range of salinity levels (Cho et al., 2010). However, postmortem studies using transgenic marine medaka have largely focused on the responses to natural estrogen, estradiol-17 $\beta$, whereas the transgenic expression pattern related to synthetic estrogenic derivatives (e.g., EE2) has yet to be characterized in detail. In line with our longterm goal of developing a multilevel integrated approach to address estrogen responses using transgenic marine medaka models, the objective of the present study was to examine the EE2-induced expression patterns of the chgH:rfp transgene in marine medaka.

\section{Materials and Methods}

\section{Transgenic fish strain}

The transgenic marine medaka individuals used in the present study were F4 generation transgenic individuals carrying the red fluorescent protein gene $(r f p)$ driven by an endogenous choriogenin $\mathrm{H}(\mathrm{chg} H)$ promoter. The transgene integration and transmission patterns as well as the estradiol$17 \beta$ (E2)-mediated expression profiles of this transgenic line (TG\#038) are provided in our previous report (Cho et al., 2013). Hemizygous offspring were propagated by mat- ing transgenic males with non-transgenic females, and stable germline transmission of the transgene resembling the pattern of Mendelian single-gene inheritance was confirmed by PCR typing of the transgene with a random sample of progeny ( $n$ $\geq 27$ ) obtained from each mating group. Larval DNA samples were subjected to PCR amplification of a 258-bp internal segment of chgH:rfp transgene using the primer pairs OD-TG 1F: 5'- GAGAGGTGGAGTTTGAAGAG -3' and OD-TG 1R: 5'-ACCTTCAGCTTCACGGTGT - $3^{\prime}$ under the following thermal cycling conditions: 30 cycles at $94^{\circ} \mathrm{C}$ for $20 \mathrm{~s}, 60^{\circ} \mathrm{C}$ for 20 $\mathrm{s}$, and $72^{\circ} \mathrm{C}$ for $20 \mathrm{~s}$, with an initial denaturation step at $94^{\circ} \mathrm{C}$ for $3 \mathrm{~min}$.

\section{Laboratory breeding and preparation of larval samples for exposure}

To obtain hemizygous transgenic larvae for the exposure studies, group mating was carried out between five-monthold transgenic males (half-siblings; $n=10$ ) and same-aged non-transgenic, wild-type females $(n=30)$. A temperature of $25 \pm 1{ }^{\circ} \mathrm{C}$ was maintained throughout the mating experiment, and dissolved oxygen was maintained at $6 \pm 1 \mathrm{ppm}$. Salinity was adjusted to $10 \mathrm{ppt}$ using synthetic sea salt (Kent Marine, Georgia, USA). The fish were fed with a commercially available artificial diet (500 $\mu \mathrm{m}$ in diameter) for flounder larvae (Woosung Feed Corp., Daejeon, Korea). The daylight cycle was 14L:10D. The spawned and fertilized eggs were collected daily at 9:00 am, and placed in an incubator until hatching. The temperature and salinity of the incubator were the same as those of the spawning tanks. Hatched larvae from each embryo batch were also collected daily, and hatchlings from the same day were transferred to the same net cage (rectangular cage of 3-L capacity) installed in a 100 L-tank in which the salinity was maintained at $10 \mathrm{ppm}$. After yolk sac absorption (48 $\mathrm{h}$ after hatching), the larvae were fed with $100-\mu \mathrm{m}$ artificial powder for flounder larvae (Woosung Feed Corp.).

\section{EE2 exposure with different age groups of trans- genic hatchlings}

To examine the effects of the initial treatment duration on the success of transgene induction, hatchlings collected at 0 , $1,2,3,6$, and 9 days post-hatching (DPH) were exposed to $500 \mathrm{ng} / \mathrm{L}$ (nominal concentration) of EE2 for 3 days. The EE2 used in this study was analytical grade reagent purchased from a commercial supplier (Sigma-Aldrich, St Louis, MO, USA). For exposure, larvae $(n=36)$ from each age group were transferred to a $3-\mathrm{L}$ rectangular glass container containing $2.5-\mathrm{L}$ water at $10 \mathrm{ppt}$. The final concentration of EE2 $(500 \mathrm{ng} / \mathrm{L})$ for each tank was adjusted using a stock solution of EE2 (50 ng/ $\mathrm{mL}$ in $50 \%$ ethanol). To prepare non-exposed control groups, larvae $(n=36)$ from each age group were treated identically to the EE2-exposed groups but without the hormone, and were treated with the same concentration of ethanol (final 
concentration $=0.0005 \%)$. At 3 days post-exposure $(\mathrm{DPE})$, the occurrence of RFP signal in the larvae belonging to each group was examined with fluorescence microscopy using an AZ100 fluorescence microscope equipped with NIS-Elements BR image analysis software (Nikon Corporation Instruments Co., Japan). To estimate the incidence of RFP expression as a percentage of the number of fish in each treatment group, the RFP-negative larvae from each group were subjected to PCR typing of the chgH:rfp transgene (see above). Triplicate exposure experiments were conducted in an independent fashion.

\section{Experimental exposure to high concentrations of EE2}

Due to the age-dependence of the frequency of transgene induction, transgenic hatchlings of the same age, 7 days, were exposed to various concentrations of EE2 up to $1,000 \mathrm{ng} / \mathrm{L}$. The larvae (approximately 40 fish per group) were exposed to 0 (non-exposed), 200, 400, 600, 800 or 1,000 ng/mL EE2 for 3 and 6 days. All tank conditions including temperature and salinity were the same as those described above. During the exposure period, $50 \%$ of the water was exchanged daily and the hormone was simultaneously replaced with fresh hormone. As in the procedure described above, PCR screening of the transgene was conducted with RFP-negative fish to estimate the frequency of transgene expression. Duplicate exposure tests were carried out.

\section{Experimental exposure to low concentrations of EE2}

To examine the potential for detecting waterborne EE2 of dose strengths close to environmentally relevant concentrations, transgenic larvae were exposed to low doses of EE2. Five or six-day-old transgenic larvae were exposed to EE2 at dose levels of 0 (non-exposed control), 25, 50, and $100 \mathrm{ng} / \mathrm{L}$. Approximately 48 larvae per group were incubated in a $10-\mathrm{L}$ rectangular tank containing $8-\mathrm{L} 10$-ppt water at $25^{\circ} \mathrm{C}$. During exposure, the fish were fed a $100-\mu \mathrm{m}$ powder diet for flounder as above and the daily exchange rate of hormone-supplemented water was $30 \%$. From $24 \mathrm{~h}$ after exposure, the individual fish from each group were examined daily for the occurrence of RFP signals using fluorescence microscopy. At the end of the exposure period, the larvae were subjected to PCR transgene screening to estimate the efficiency of transgene induction in each group.

\section{EE2 exposure under different salinity conditions}

The effects of salinity on the EE2-mediated RFP expression were examined in the transgenic larvae. At $2 \mathrm{DPH}$, the hatchlings in the 10-ppt tank were acclimated to one of three salinities $(0,15$ and $30 \mathrm{ppt})$ according to the procedures described in our previous work (Cho et al., 2010). After five days of ac- climation at each salinity level, 7-day-old larvae $(n=40 \sim 48)$ were exposed to EE2 (100 ng/L) for 1 week. At 7 DPE, individual fish from each group were examined with fluorescence microscopy for the expression of RFP signals. Other exposure conditions, including hormone renewal, temperature and larval feeding, were the same as those described above. Two replicate examinations were carried out for each salinity group.

\section{Results and Discussion}

\section{Effects of larval age on the success of transgene expression during EE2 exposure}

Under the microscopy conditions used in this study, none of non-transgenic larvae showed any notable red fluorescence signal, with the exception of minute background fluorescence around the eyes (not the lens). Also, no transgenic larvae displayed apparent RFP expression under non-exposed conditions, including the transgenic larvae in the ethanol-treated control groups. However, among the groups exposed to EE2, the transgenic larvae displayed a strong RFP signal in their livers (Fig. 1). The liver-specific induction of RFP signals was consistent in all the RFP-positive larvae, indicating that the liver-dominant regulation of choriogenin $\mathrm{H}$ promoter was well manifested in transgenic larvae in response to exogenously introduced EE2. However, in a previous study, it was reported that choriogenin $\mathrm{H}$ gene transcripts were detectable in several non-liver organs of adult marine medaka (Lee et al., 2012b), which differs from our findings of liver-exclusive appearance of RFP signals in transgenic larvae. Hence, further studies exploring the regulation mechanisms of the choriogenin $\mathrm{H}$ gene in non-liver tissues throughout the life cycle of marine medaka are necessary.

Although EE2-mediated induction of RFP was apparent in transgenic larvae, the frequency of RFP expression was significantly affected by the larval age (i.e., initial exposure timing) (Fig. 1). Based on the PCR typing, all the tested groups showed similar transgenic incidence of close to $50 \%$, resembling the Mendelian single gene inheritance, indicating that hemizygous transgenic propagation was achieved as expected (Kim et al., 2004). However, the rate of RFP expression varied significantly, ranging from $24 \%$ to $100 \%$ among age groups, and the frequency of the RFP signal increased with increasing age up to $9 \mathrm{DPH}$. When just-hatched larvae were exposed to EE2, less than $25 \%$ of the transgenic larvae expressed RFP in response to EE2. However, this low frequency increased significantly when older larvae were exposed to the same concentration of EE2. As a result, almost $100 \%$ of the transgenic larvae showed RFP expression for EE2 exposures beginning at $6 \mathrm{DPH}$ and $9 \mathrm{DPH}$. Similar findings have been reported with exposure to the natural hormone E2 (Cho et al., 2013), suggesting that stimulation of the transgene in response to natural and synthetic estrogens does not differ significantly in 

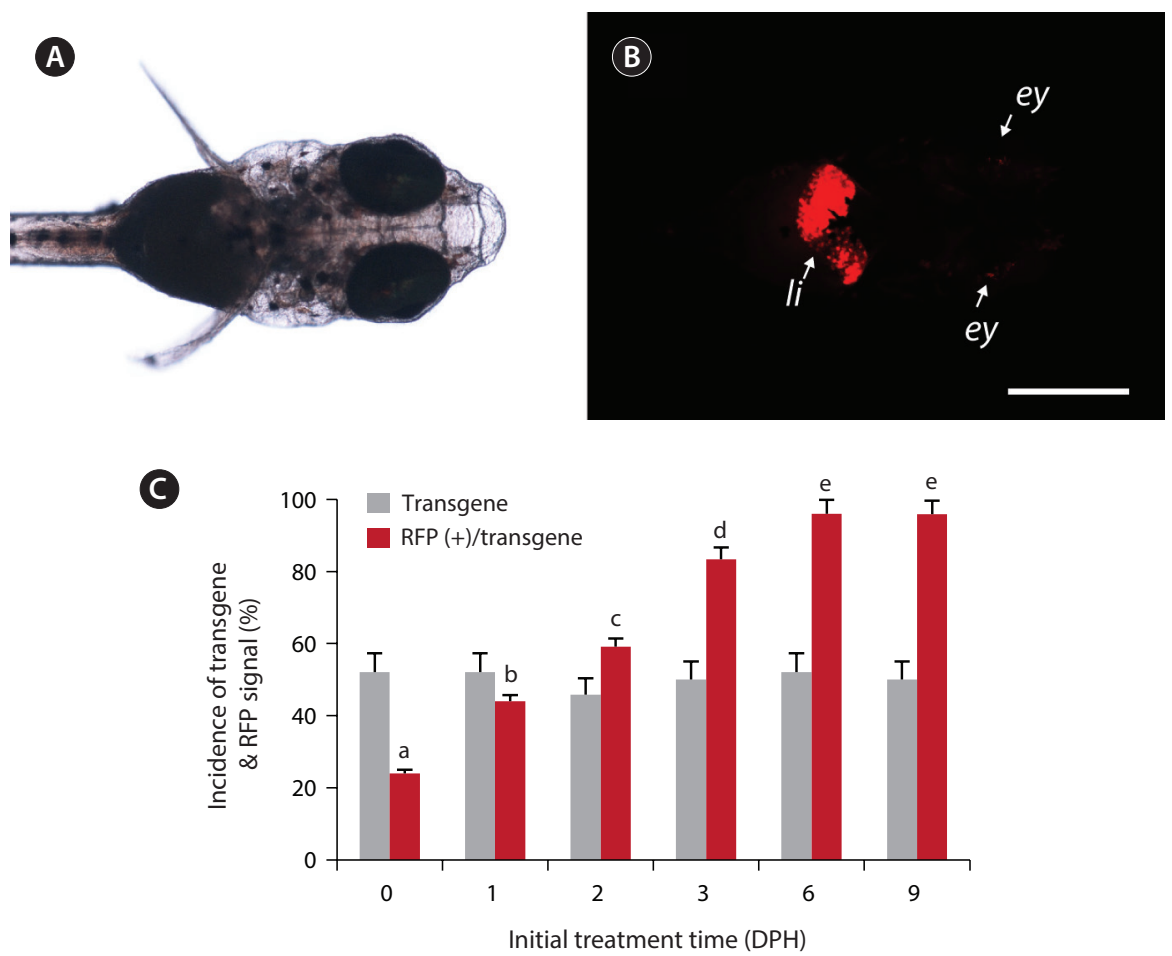

Fig. 1. Effects of larval ages on the efficiency of EE2-mediated induction of RFP signals in transgenic marine medaka Oryzias dancena. A) An RFP-positive transgenic larva (belonging to the $6 \mathrm{DPH}$ age) photographed under light-field microscopic condition. B) Dark field image of $A$ ) showing the liver-specific induction of RFP signals. Eyes (ey) and RFP-expressing liver (li) are indicated by arrow. Bar is $1 \mathrm{~mm}$. C) Incidence of RFP-positive transgenic fish in relation with the initial exposure time. Incidence of transgene was calculated based on the PCR typing of transgene. The efficiency of RFP expression was estimated as a percentage of transgenics. All the groups exhibited the similar frequency of transgenics close to $50 \%(P>0.05)$ whereas the RFP induction in response to EE2 exposure ( $500 \mathrm{ng} / \mathrm{L}$ for 3 days) was differentially presented depending upon the initial treatment time (larval age from 0 to 9 days post hatching; $\mathrm{DPH})$. The means with different letters (for incidence of RFP induction) were significantly different each other based on ANOVA followed by Duncan's multiple ranged tests $(P<0.05)$.

the early larval stages. Although the absolute levels of RFP incidence are clearly affected by estrogen type and treatment dose strength, our data suggest that transgenic functionality is not fully achieved in the very early developmental stages and, thus, the use of transgenic larvae of a specific age is recommended for detection of waterborne estrogenicity.

Table 1. Incidence of RFP-positive transgenics during experimental exposures to different concentrations of EE2 (17a-ethinylestradiol) for 3 or 6 days $^{*}$

\begin{tabular}{ccc}
\hline \multirow{2}{*}{ EE2 dose (ng/L) } & \multicolumn{2}{c}{ Exposure period } \\
\cline { 2 - 3 } & Day 3 & Day 6 \\
\hline 0 & 0.0 & 0.0 \\
200 & 6.7 & 100.0 \\
400 & 63.4 & 98.7 \\
600 & 73.5 & 100.0 \\
800 & 93.4 & 100.0 \\
1,000 & 100.0 & 100.0 \\
\hline
\end{tabular}

"Incidence of RFP-expressing fish for each group was estimated as a percentage of transgenics based on the PCR typing of transgene. Each incidence is the average value of duplicated examinations.

\section{Semi-quantitative expression of transgenic RFP during exposure using high concentrations of EE2}

During EE2 exposure with concentrations ranging from 200 to $1000 \mathrm{ng} / \mathrm{L}$, the frequency of RFP expression was, in general, positively related to EE2 concentration at 3 DPE (Table 1). The $200 \mathrm{ng} / \mathrm{L}$-treated group showed less than 10\% incidence of RFP expression, which increased to $63 \%$ at 400 $\mathrm{ng} / \mathrm{L}$, and further increased with higher EE2 doses to 90$100 \%$. However, the observed dose dependency decreased at 6 DPE, for which most exposed groups showed $100 \%$ expression of RFP. The dose-dependent appearance of RFP-positive larvae at 3 DPE may reflect differential EE2 uptake by the fish during the early phase of exposure. On the other hand, the plateau of RFP induction at 6 DPE may be related to the rapid bioaccumulation of EE2 during the extended exposure period, which is consistent with previous reports that EE2 is highly resistant to biodegradation (Zhang et al., 2006; Aris et al., 2014). In addition to the induction efficiency, fluorescence microscopy analysis showed that the RFP intensity in the livers of the transgenic larvae was also positively correlated with EE2 dose, particularly at 3 DPE (Fig. 2). Under the assay con- 

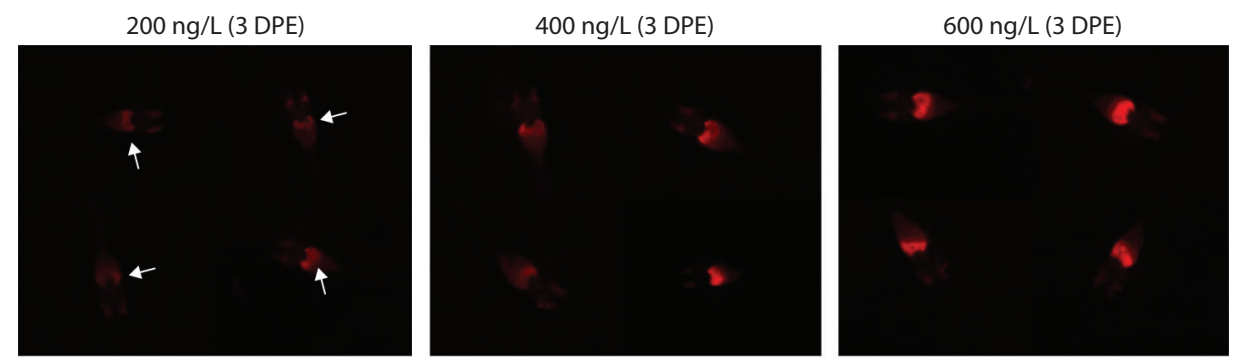

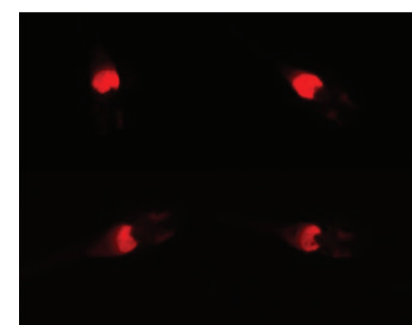

$800 \mathrm{ng} / \mathrm{L}(3 \mathrm{DPE})$

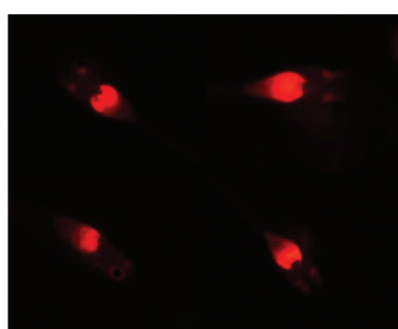

$1000 \mathrm{ng} / \mathrm{L}$ (3 DPE)

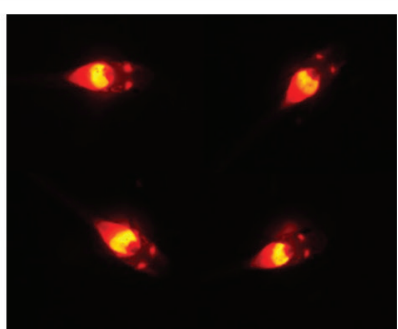

$400 \mathrm{ng} / \mathrm{L}(6 \mathrm{DPE})$

Fig. 2. Fluorescence microscopic images showing the representative RFP-positive transgenics exposed to different concentrations of EE2. Images were obtained at 3 or 6 days post exposure (DPE). At 3 DPE, the intensities of RFP signals induced in transgenic livers were positively related with concentrations of EE2. However, at 6 DPE, almost transgenics showed the saturation-like RFP signals irrespective of concentrations of EE2 (only the fish from $400 \mathrm{ng} /$ L-exposed group are shown). The RFP-positive livers of the fish exposed with $200 \mathrm{ng} / \mathrm{L}$ for 3 days are indicated by arrows.

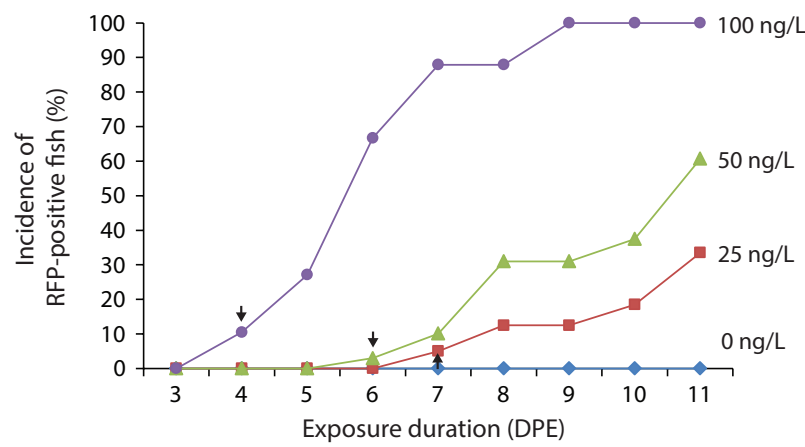

Fig. 3. Time course patterns of the cumulative incidence of RFP-positive transgenics during experimental exposure to EE2 (0 -100 ng/L). Incidence of RFP signal was calculated as a percentage of transgenics. The first appearance of RFP-positive fish in each EE2-exposed group is indicated by an arrow. DPE, days post exposure.

ditions used in our study, the dose-dependent increase in RFP intensity in the livers of the transgenic larvae was apparent at 3 DPE, although there were also variations among individuals within a given exposure dose, suggesting that transgenic detection of EE2 could be performed, in at least a semi-quantitative manner (Cho et al., 2013). However, at 6 DPE, most, but not all, transgenic larvae showed a tendency toward saturation of fluorescence regardless of EE2 concentration. Hence, further studies are required to compare the actual tissue burden (i.e., absolute amount of EE2 in tissues) with the level of RFP expression to improve quantitative detection of EE2 using transgenic fish larvae.

\section{Time course expression patterns of transgenic RFP during exposure to low concentrations of EE2}

Transgenic detection of lower concentrations of EE2 was also possible using chgH:rfp-transgenic marine medaka; however, the minimum exposure period needed for the RFP induction was inversely related to EE2 concentration (Fig. 3). For the $25 \mathrm{ng} / \mathrm{L}$ group, the first occurrence of the RFP signal was observed at 7 DPE with an incidence of only 5\%. Expression gradually increased with extended exposure duration such that approximately $33 \%$ of the transgenic larvae successfully expressed RFP in response to EE2 at 11 DPE. In the $50 \mathrm{ng} / \mathrm{L}$ group, the onset of the RFP signal was observed at 6 DPE (in only $3 \%$ of the transgenics), and increased to $31 \%$ and $61 \%$ at 8 and 11 DPE, respectively. Finally, the larval group exposed to $100 \mathrm{ng} / \mathrm{L}$ began to exhibit induced RFP expression at $4 \mathrm{DPE}$ with an incidence of $10 \%$. Subsequently, the incidence of RFP induction increased rapidly with exposure duration, reaching $100 \%$ at 9 DPE. In all three exposed groups, the RFP expression intensified with exposure duration (photographs not shown). Our findings suggest that $\operatorname{ch} H$ :rfp-transgenic marine medaka systems can be applied to detect EE2 where doses are close to environmentally relevant concentrations, although the detection limit using this transgenic system could be further explored using lower concentrations and prolonged exposure. Considering that environmentally realistic doses of EE2 reported in previous exposure studies with different fish species were less than $50 \mathrm{ng} / \mathrm{L}$ (Shved et al., 2008; Hirakawa et al., 2012; Lee et al., 2014), the transgenic approach developed in the present study could be applied to the detection of EE2 in aquatic environments with acceptable sensitivity. 

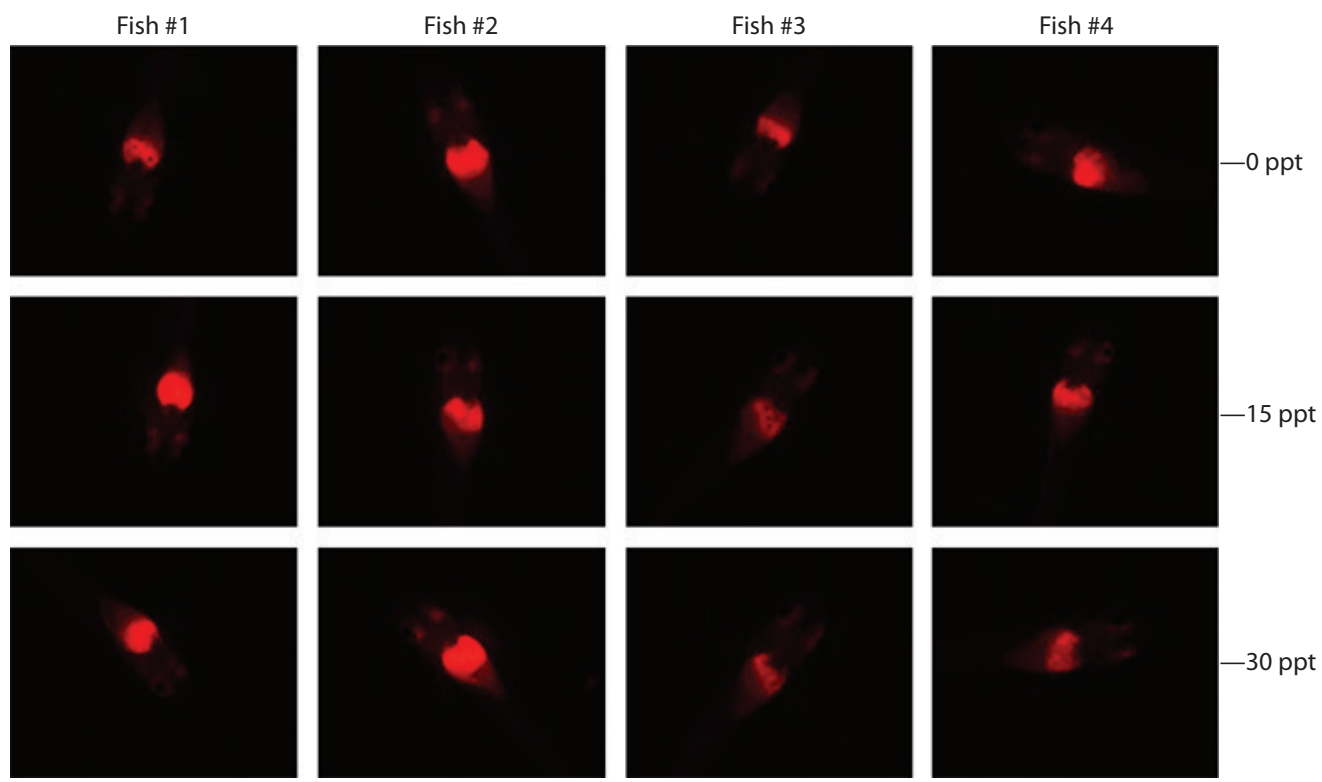

Fig. 4. Representative fluorescent images to show the RFP-positive transgenic larvae (four selected individuals per salinity group) exposed to $100 \mathrm{ng} /$ L EE2 for 1 week under different salinity conditions (0,15 and $30 \mathrm{ppt})$. Although variations of RFP intensities among individuals were observable in each salinity group, no apparent difference in the strength of RFP expression was found among salinity levels tested.

\section{EE2-induced transgene expression under differ- ent salinity conditions}

Under the exposure conditions used in the present study, transgenic expression at the phenotypic level in response to EE2 exposure did not differ among salinity groups in either the intensity or incidence of the RFP signal acquired in the transgenic larvae. After 1 week of exposure, all salinity groups $(0,15$, and $30 \mathrm{ppt})$ displayed a high incidence of RFP induction $(89.5 \%$ for $0 \mathrm{ppt}, 91.3 \%$ for $15 \mathrm{ppt}$, and $89.5 \%$ for $30 \mathrm{ppt}$ ). The RFP intensity induced in the livers of the transgenic larvae assessed by fluorescence microscopy was also similar among all salinity groups, although some inter-individual variation was observed within each group (Fig. 4). These findings suggest that the chgH:rfp transgenic marine medaka system could be applied for detection of waterborne EE2 at a wide range of salinities, which is in agreement with our previous observations with E2 exposure (Cho et al., 2013). However, although the general applicability of this transgenic approach to variable salinities could be established, a more accurate quantitative comparison of the effects of salinity might also be needed since salinity conditions have been reported to affect the EE2 uptake by the euryhaline teleost, killifish Fundulus heteroclitus (Blewett et al., 2013). In addition, it has been reported that marine medaka fry acclimated to either freshwater or seawater possessed differential basal levels of choriogenin $\mathrm{H}$ transcripts (Lee et al., 2012b). This reinforces the need for further studies of differences in the inducibility of transgene and endogenous gene expression during EE2 exposure under different salinity conditions.
Our choriogenin $\mathrm{H}$ promoter-driven reporter transgenic system developed in euryhaline marine medaka represents a straightforward approach to the detection of various concentrations of waterborne EE2, a widespread estrogenic pollutant in aquatic environments. Based on its acceptable sensitivity, this transgenic approach using marine medaka offers significant potential for detecting EE2 in a semi-quantitative and environmentally relevant manner. To build upon this study, detailed investigation of both the biotic and abiotic factors affecting the sensitivity and efficiency of transgenic expression should be carried out.

\section{Acknowledgments}

This study was supported by the grant from Ministry of Oceans and Fisheries, Korea.

\section{References}

Aris AZ, Shamsuddin AS, Praveena SM. 2014. Occurrence of $17 \alpha$-ethynylestradiol (EE2) in the environment and effect on exposed biota: a review. Environ Intl 69, 104-119.

Blewett T, MacLatchy DL, Wood CM. 2013. The effects of temperature and salinity on $17 \alpha$-ethinylestradiol uptake and its relationship to oxygen consumption in the model euryhaline teleost (Fundulus heteroclitus). Aquat Toxicol 127, 61-71.

Chen H, Hu J, Yang J, Wang Y, Xu H, Jiang Q, Gong Y, Gu Y, Song H. 2010. Generation of a fluorescent transgenic zebrafish for detection 
of environmental estrogens. Aquat Toxicol 96, 53-61.

Cho YS, Kim DS, Nam YK. 2013. Characterization of estrogen-responsive transgenic marine medaka Oryzias dancena germlines harboring red fluorescent protein gene under the control by endogenous choriogenin H promoter. Transgen Res 22, 501-517.

Cho YS, Lee SY, Kim DS, Nam YK. 2010. Tolerance capacity to salinity changes in adult and larvae of Oryzias dancena, a euryhaline medaka. Kor J Ichthyol 22, 9-16.

Filby AL, Thorpe KL, Maack G, Tyler CR. 2007. Gene expression profiles revealing the mechanisms of anti-androgen- and estrogeninduced feminization in fish. Aquat Toxicol 81, 219-231.

Harding LB, Schultz IR, Goetz GW, Luckenbach JA, Young G, Boetz FW, Swanson P. 2013. High-throughput sequencing and pathway analysis reveal alteration of the pituitary transcriptome by $17 \alpha$-ethynylestradiol (EE2) in female coho salmon Oncorhynchus kisutch. Aquat Toxicol 142-143, 146-163.

Hirakawa I, Miyagawa S, Katsu Y, Kagmi Y, Tatarazako N, Kobayashi T, Kusano T, Mizutani T, Ogino Y, Takeuchi T, Ohta Y, Iguchi T. 2012. Gene expression profiles in the testis associated with testis-ova in adult Japanese medaka (Oryzias latipes) exposed to 17a-ethynylestradiol. Chemosphere 87, 668-674.

Kim DS, Kim BS, Lee SJ, Park IS, Nam YK. 2004. Comparative analysis of inherited patterns of the transgene in transgenic mud loach Misgurnus mizolepis lines carrying the CAT reporter gene. Fish Sci 70, 201-210.

Kurauchi K, Hirata T, Kinoshita M. 2008. Characteristics of ChgH-GFP transgenic medaka lines, an in vivo estrogenic compound detection system. Mar Pollut Bull 57, 441-444.

Lange R, Hutchinson TH, Croudace CP, Siegmund F, Schweinfurth H, Hampe P, Panter GH, Sumpter JP. 2001. Effects of the synthetic estrogen 17 1 -ethinylestradiol on the life-cycle of the fathead minnow (Pimephales promelas). Environ Toxicol Chem 20, 12161227.
Lee O, Takesono A, Tada M, Tyler CR, Kudoh T. 2012a. Biosensor zebrafish provide new insights into potential health effects of environmental estrogens. Environ Health Perspect 120, 990-996.

Lee PY, Lin CY, Chen TH. 2014. Environmentally relevant exposure of $17 \alpha$-ethinylestradiol impairs spawning and reproductive behavior in the brackish medaka Oryzias melastigma. Mar Pollut Bull 85, 338-343.

Lee SY, Kim DS, Nam YK. 2012b. Gene structure and estrogen-responsive mRNA expression of a novel choriogenin $\mathrm{H}$ isoform from a marine medaka Oryzias dancena. Fish Aqaut Sci 15, 221-231.

Nadzialek S, Pigneur LM, Weron B, Kestemont P. 2010. Bcl-2 and Caspase 3 mRNA levels in the testes of gudgeon, Gobio gobio, exposed to $17 \alpha$-ethinylestradiol (EE2). Aquat Toxicol 98, 304-310.

Petersen K, Fetter E, Kah O, Brion F, Scholz S, Tollefsen KE. 2013. Transgenic (cyp19alb-GFP) zebrafish embryos as a tool for assessing combined effects of oestrogenic chemicals. Aquat Toxicol 138-139, 88-97.

Shved N, Berishvili G, Baroiller JF, Segner H, Reinecke M. 2008. Environmentally relevant concentrations of $17 \alpha$-ethinylestradiol (EE2) interfere with the growth hormone (GH)/insulin-like growth factor (IGF)-I system in developing bony fish. Toxicol Sci 106, 93-102.

Shved N, Berishvili G, Hausermann E, D'Cotta H, Baroiller JF, Eppler E. 2009. Challenge with $17 \alpha$-ethinylestradiol (EE2) during early development persistently impairs growth, differentiation, and local expression of IGF-I and IGF-II in immune organs of tilapia. Fish Shellfish Immunol 26, 524-530.

Teng Q, Ekman DR, Huang W, Collette TW. 2013. Impacts $17 \alpha$-ethynylestradiol exposure on metabolite profiles of zebrafish (Danio rerio) liver cells. Aquat Toxicol 130-131, 184-191.

Zhang X, Chen P, Wu F, Deng N, Liu J, Fang T. 2006. Degradation of $17 \alpha$-ethinylestradiol in aqueous solution by ozonation. J Hazard Mat 133B, 291-298. 